\title{
La Freccia Rossa: an IR-dark cloud hosting the Milky Way intermediate-mass black hole candidate
}

\author{
Vikram Ravi, ${ }^{1 \star}$ Harish Vedantham ${ }^{1}$ and E. Sterl Phinney ${ }^{2}$ \\ ${ }^{1}$ Cahill Center for Astronomy and Astrophysics MC 249-17, California Institute of Technology, Pasadena, CA 91125, USA \\ ${ }^{2}$ Theoretical Astrophysics MC 350-17, California Institute of Technology, Pasadena, CA 91125, USA
}

Accepted 2018 April 29. Received 2018 April 09; in original form 2017 October 10

\begin{abstract}
The dynamics of the high-velocity compact molecular cloud CO-0.40-0.22 have been interpreted as evidence for a $\sim 10^{5} \mathrm{M}_{\odot}$ black hole within $60 \mathrm{pc}$ of Sgr A*. Recently, Oka et al. have identified a compact millimetre-continuum source, CO-0.40-0.22*, with this candidate black hole. Here we present a collation of radio and infrared data at this location. Australia Telescope Compact Array constraints on the radio spectrum, and the detection of a mid-infrared counterpart, are in tension with an Sgr A*-like model for CO-0.40-0.22* despite the comparable bolometric to the Eddington luminosity ratios under the intermediate-mass black hole interpretation. A protostellar-disc scenario is, however, tenable. CO-0.40-0.22(*) is positionally coincident with an arrowhead-shaped infrared-dark cloud (which we call the Freccia Rossa). If the $V_{\mathrm{LSR}} \approx 70 \mathrm{~km} \mathrm{~s}^{-1}$ systemic velocity of CO-0.40-0.22 is common to the entire Freccia Rossa system, we hypothesize that it is the remnant of a high-velocity cloud that has plunged into the Milky Way from the Galactic halo.
\end{abstract}

Key words: black hole physics-radiation mechanisms: general-stars: formation-ISM: clouds - dust, extinction - Galaxy: halo.

\section{INTRODUCTION}

Oka et al. (2017) present evidence for a $\sim 10^{5} \mathrm{M}_{\odot}$ intermediatemass black hole (IMBH) lurking within the central $60 \mathrm{pc}$ of the Milky Way. The first hint of this object was the discovery of a compact molecular cloud with an unusually high line-of-sight velocity, and velocity dispersion (C0-0.40-0.22; Oka et al. 2012; Tanaka et al. 2014). Such clouds (Oka et al. 2007) are common within the Central Molecular Zone (CMZ) of the Galaxy, and have few parsec sizes and velocity spreads of a few tens of $\mathrm{km} \mathrm{s}^{-1}$. Many of these clouds lack unambiguous sources of the high internal velocities, and their origins have been attributed to shocks caused by unseen supernovae (Oka et al. 2007), and turbulence in colliding clouds or expanding molecular shells (Tanaka et al. 2014). However, with an internal kinetic energy of $10^{49.7} \mathrm{erg}$ (Oka et al. 2016) and its lack of an expanding velocity structure, $\mathrm{C} 0-0.40-0.22$ appears inconsistent with these explanations. Its dynamics are consistent with a slingshot from an unseen $\sim 10^{5} \mathrm{M}_{\odot}$ compact object (Oka et al. 2016). ALMA images with $\sim 1$ arcsec resolutionat 231 and $266 \mathrm{GHz}$ revealed a point-like continuum source within C0-0.40-0.22 (Oka et al. 2017). This source (C0-0.40-0.22*) is interpreted by Oka et al. (2017) as an analogue of the Galactic-centre black hole Sgr A*.
IMBHs with masses between $10^{2}$ and $10^{6} \mathrm{M}_{\odot}$ are predicted to exist in the present-day Universe as a general consequence of the supermassive black hole seed formation (Volonteri 2010; Greene 2012). The likely numerous historical minor mergers of the Milky Way with companion dwarf galaxies (Gilmore, Wyse \& Norris 2002; van Loon et al. 2003) may plausibly have led to an IMBH sinking to the centre of the Milky Way potential well (Colpi 2014). If $\mathrm{C} 0-0.40-0.22 *$ is such an IMBH, it would provide an important clue to the merger history of the Milky Way, and provide a new site for the study of the interaction between massive black holes and their environments.

Thus motivated, we use the wealth of multiwavelength data available at the position of $\mathrm{C} 0-0.40-0.22 *$ to better characterize this candidate IMBH and its environment. We collated archival $24.6 \mathrm{GHz}$ continuum observations from the Australia Telescope Compact Array (ATCA), mid-IR observations from the Spitzer Space Telescope, and Ks-band observations from the Visible and Infrared Survey Telescope for Astronomy (VISTA), and augmented these with new continuum observations from the ATCA. In Section 2, we present and analyse measurements of the spectral energy distribution (SED) of $\mathrm{C} 0-0.40-0.22 *$. Then, in Section 3, we characterize the environment of C0-0.40-0.22*. We discuss our results in Section 4, and conclude in Section 5.

^E-mail: v.vikram.ravi@gmail.com 


\section{THE ALMA POINT-SOURCE CO-0.40-0.22*}

Oka et al. (2017) detected C0-0.40-0.22* in ALMA continuum observations as an unresolved source (synthesized beam of 1.35 arcsec $\times 0.55$ arcsec), with flux densities of $8.38 \pm 0.34 \mathrm{mJy}$ at $231 \mathrm{GHz}$, and $9.91 \pm 0.74 \mathrm{mJy}$ at $266 \mathrm{GHz}$. The implied spectral index was $\alpha=1.18 \pm 0.65$. $^{1}$ Oka et al. (2017) also presented a $3 \sigma$ upper limit on the $1-7 \mathrm{keV}$ flux of $1.4 \times 10^{-14} \mathrm{erg} \mathrm{s}^{-1} \mathrm{~cm}^{-2}$.

We obtained centimetre-wavelength observations of CO-0.40$0.22 *$ with the ATCA on 2017 September 8 (13:50ut). The six $22 \mathrm{~m}$ dishes of the array were arranged in the H168 configuration, with five dishes in a compact ' $\mathrm{T}$ ' with a maximum baseline of $192 \mathrm{~m}$, and the sixth dish located approx. $4400 \mathrm{~m}$ from the ' $\mathrm{T}$ '. Full-Stokes data were recorded with the Compact Array Broad-band Backend (CABB; Wilson et al. 2011) in a standard 64M-32k continuum setup, in sidebands centred on $32.5 \mathrm{GHz}$ and $36 \mathrm{GHz}$. The flux density scale was set using a 10- min observation of PKS 1934-638, and the receiver band passes and complex gains were calibrated using the quasar PKS 1741-312 (1.9 ${ }^{\circ}$ distant from the program source). Observing conditions were excellent, with negligible wind and $<100 \mu \mathrm{m}$ of rms atmospheric path-length variation. We were able to track the atmospheric phase variations on our longest baselines with sufficient accuracy by cycling between 2 -min observations of CO-0.40-0.22* and 40-s observations of PKS 1741-312. The pointing of each antenna was checked and corrected every $30 \mathrm{~min}$. The total time spent observing CO-0.40-0.22* was $61 \mathrm{~min}$.

We reduced and calibrated our data using standard techniques with the MIRIAD software (Sault, Teuben \& Wright 1995). To search for unresolved emission from CO-0.40-0.22*, we made a multifrequency synthesis image of our data from both sidebands simultaneously with uniform weighting. The full width at half-maximum (FWHM) of the synthesized beam was $0.99 \operatorname{arcsec} \times 0.23$ arcsec. No source was detected at the position of $\mathrm{CO}-0.40-0.22 *$; we set a $3 \sigma$ upper limit on its $34.25 \mathrm{GHz}$ flux density of $0.285 \mathrm{mJy}$.

We also analysed an archival mid-IR data from the Spitzer IR Array Camera (IRAC) obtained as part of the Galactic Legacy Infrared Mid-Plane Survey Extraordinaire (GLIMPSE) program (Churchwell et al. 2009). We downloaded calibrated image cutouts in all the four IRAC bands $(3.6,4.5,5.8$, and $8.0 \mu \mathrm{m})$ from the IRSA Cutouts Service $^{2}$ at the position of CO-0.40-0.22*, rendered with the original 1.2 arcsec pixels. A faint point-like source was evident at the position of CO-0.40-0.22* in the 3.6 and $4.5 \mu \mathrm{m}$ images. As the field was exceedingly crowded, complicated by diffuse emission, and because the source was 9 arcsec froma brighter star, we estimated the flux density of the source by summing the signal within a 1-pixel radius aperture centred on the position of CO-0.40$0.22 *$, and subtracted the mean background level from a 1-pixel annulus surrounding the aperture. The resulting flux densities were $0.16 \pm 0.07 \mathrm{mJy}$ at $3.6 \mu \mathrm{m}$, and $0.21 \pm 0.09 \mathrm{mJy}$ at $4.5 \mu \mathrm{m}$. The small aperture, in combination with the unknown interstellar extinction towards $\mathrm{CO}-0.40-0.22 *$, implies that these measurements are lower limits.

This mid-IR source was also detected in the Ks-band in a deep stack of images from the VISTA Variables in the Via Lactea survey (Minniti et al. 2010). We obtained pipeline-processed, calibrated, and stacked data from the VISTA Science Archive (Cross et al. 2012), and performed standard aperture photometry. The measured flux density was $0.17 \pm 0.08 \mathrm{mJy}$. The unknown extinction again

\footnotetext{
${ }^{1}$ The spectral index, $\alpha$, is defined for a flux-density spectrum $F_{v} \propto \nu^{\alpha}$.

${ }^{2} \mathrm{http} / / /$ irsa.ipac.caltech.edu/applications/Cutouts/spitzer.html
}

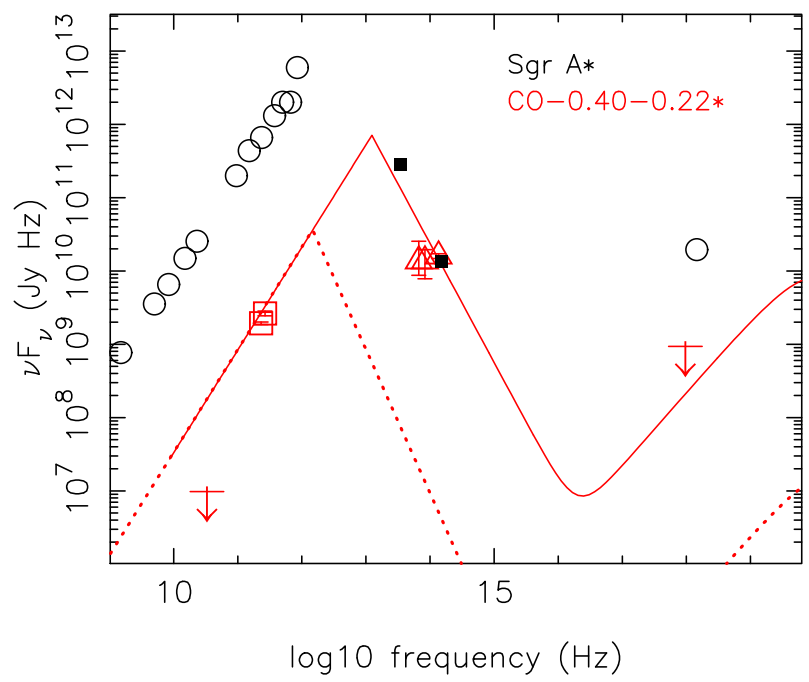

Figure 1. The broad-band SED of the IMBH candidate CO-0.40-0.22* (red arrows, open squares, and triangles), in comparison with the quiescent SED of Sgr A* (black circles and solid squares). The 231 and $266 \mathrm{GHz}$ detections of CO-0.40-0.22* (red open squares) and the X-ray $3 \sigma$ upper limit (red arrow) were presented by Oka et al. (2017). The $34.25 \mathrm{GHz} 3 \sigma$ upper limit (red arrow) and the IR detections (red triangles) were obtained through the analysis presented herein. The quiescent SED of Sgr A* was compiled from the references listed in fig. 1 of Genzel et al. (2010). Detections of Sgr $\mathrm{A} *$ are shown as open black circles, and upper limits are indicated by the solid black squares. Neither SED has been corrected for extinction in the IR bands, or for interstellar absorption in the X-ray bands. Model ADAF SEDs for CO-0.40-0.22*, with $m=10^{5} \mathrm{M}_{\odot}$ and $m=2.5 \times 10^{4} \mathrm{M}_{\odot}$, are shown as dotted and solid curves, respectively.

implies that this is a lower limit. The full SED of CO-0.40-0.22* is presented in Fig. 1. For comparison, we show the SED of the Galactic-Centre black hole Sgr A* compiled as described in fig. 1 of Genzel, Eisenhauer \& Gillessen (2010). Images of the ATCA $34.25 \mathrm{GHz}$ non-detection and the Spitzer/IRAC and VISTA detections are shown in Fig. 2.

\subsection{A synchrotron interpretation}

Assuming the distance of Sgr A* (8.3 kpc; Gillessen et al. 2013), the bolometric luminosity of CO-0.40-0.22* is $\sim 10^{35} \mathrm{erg} \mathrm{s}^{-1}$, or eight orders of magnitude less than the Eddington luminosity of a $10^{5} \mathrm{M}_{\odot}$ IMBH. This ratio is comparable to that of Sgr A* (Genzel et al. 2010). We therefore first consider whether the SED of CO-0.40-0.22* can be explained through mechanisms analogous to those attributed to $\mathrm{Sgr} \mathrm{A} *{ }^{3}$ In its quiescent state, the peak of the Sgr A* SED can be largely explained by the cyclo-synchrotron radiation (e.g. Özel, Psaltis \& Narayan 2000; Wolfe \& Melia 2006) from thermal $\left(T_{\mathrm{e}} \sim 10^{10} \mathrm{~K}\right)$ electrons in an advection-dominated accretion flow (ADAF; e.g. Rees et al. 1982; Mahadevan 1997; Yuan, Quataert \& Narayan 2003). Some ultraviolet (UV) and X-ray flux is expected due to inverse-Compton (IC) scattering of the synchrotron emission. If CO-0.40-0.22* is an analogue of Sgr A $*$, the mid-IR detections imply that its spectral peak must be at approximately an order-of-magnitude higher frequency than Sgr A*. Furthermore, the $32-266 \mathrm{GHz}$ spectrum of CO-0.40-0.22* is harder than that of Sgr A*.

\footnotetext{
${ }^{3}$ We assume that the CO-0.40-0.22* SED is not affected by a variable
} accretion rate between epochs. 

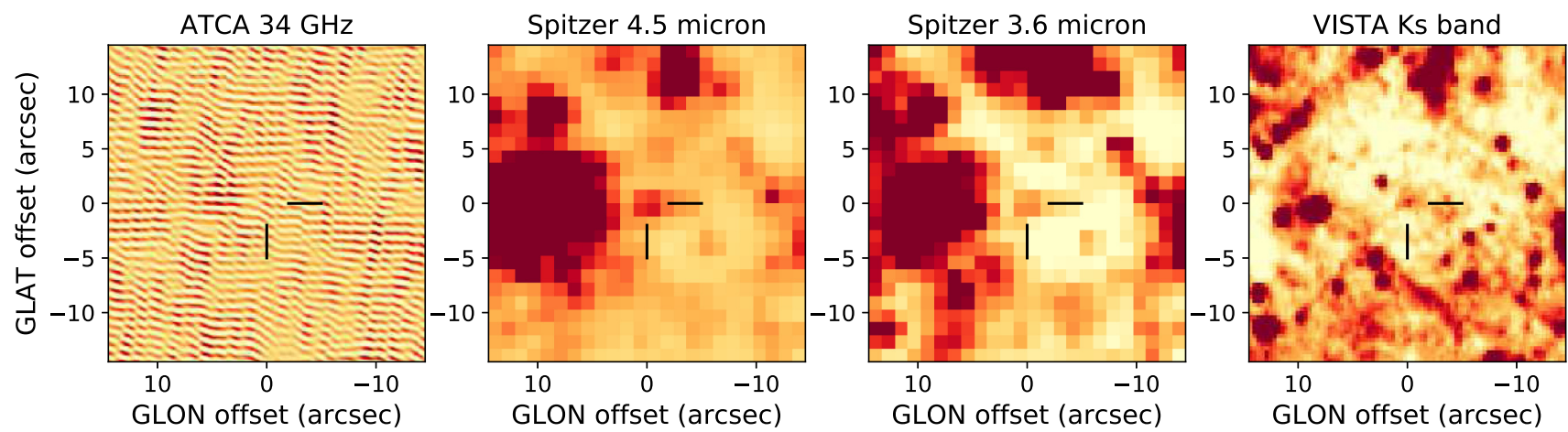

Figure 2. Images of CO-0.40-0.22* obtained with the ATCA ( $34.25 \mathrm{GHz})$, Spitzer/IRAC (4.5 and $3.6 \mu \mathrm{m})$, and VISTA (Ks band). Each image is displayed in Galactic coordinates centred on the ALMA position of CO-0.40-0.22* $(l=-0.3983, b=-0.2235)$ thatis indicated by the black lines. The colour scale in the ATCA image ranges from -1 to $+3 \sigma$.

We attempted to fit an ADAF emission model to the SED of CO-0.40-0.22* using the formulation of Mahadevan (1997). We assumed standard ADAF parameters, specified by the viscosity parameter $(\alpha=0.3)$, the magnetic-to-gas pressure ratio ( $\beta=0.5)$, the fraction of viscous heating transferred to electrons $\left(\delta=5 \times 10^{-4}\right)$, and the minimum (three gravitational radii $\left.r_{\mathrm{g}}\right)$ and maximum $\left(r_{\max }=10^{3} r_{\mathrm{g}}\right)$ radial distances from the black hole with a constant $T_{\mathrm{e}}$. The free parameters include the black hole mass, $m$, and the accretion rate in units of the Eddington rate, $\dot{m}$; an energy-balance condition fixes $T_{\mathrm{e}}$ for specific values of $m$ and $\dot{m}$. Our arguments are robust to these assumptions. We evaluate the optically thick synchrotron spectrum from the minimum frequency $v_{\min }$ (set by $r_{\max }$ ) to the peak frequency; this consists of the superposition of self-absorbed thermal cyclo-synchrotron peaks emitted at lower frequencies at larger radii. We then evaluate the IC and the thermal brehmsstrahlung emission above the SED peak.

For a given $m, \dot{m}$ is fixed by the ALMA detections of CO- $0.40-0.22 *$. For $m=10^{5} \mathrm{M}_{\odot}$, we find $\dot{m}=5 \times 10^{-7}$ and $T_{\mathrm{e}}=9.2 \times 10^{9} \mathrm{~K}$. However, as shown by the dotted curve in Fig. 1, the resulting spectral peak is far too low in frequency to explain the IR emission, and the spectrum is also inconsistent with the $34 \mathrm{GHz}$ upper limit. If we vary $m$, we find a consistent solution with $m=2.5 \times 10^{4} \mathrm{M}_{\odot}, \dot{m}=3.5 \times 10^{-5}$, and $T_{\mathrm{e}}=4.8 \times 10^{9} \mathrm{~K}$ (solid curve in Fig. 1); this solution is not unique. The resulting $\dot{m}$ is only a factor of few below the Bondi-Hoyle accretion rate for this system. The tension between our $34 \mathrm{GHz}$ limit and this model could be removed by reducing the ADAF's $r_{\max }$ by a factor of 3 , removing the outer torus's dominant contributions to the $F_{v} \propto v^{2 / 5}$ spectrum, and leaving only the steeper ( $\alpha \simeq 22 / 13$ ) quasi-blackbody from beyond $r_{\max }$. However, $r_{\max } \sim 300 r_{\mathrm{g}}$ is unnaturally low for the equalization of electron and proton temperatures to occur in the standard two-temperature ADAF models at such low accretion rates.

On the other hand, the SED of CO-0.40-0.22* is also consistent with non-thermal synchrotron emission from a relativistic jet or wind. The flat $231 \mathrm{GHz}$ to IR spectrum is consistent with the $F_{v}$ $\propto v^{1 / 3}$ optically thin synchrotron emission from relativistic electrons that are monoenergetic, or whose energy distribution has a sharp lower energy cut-off. The characteristic Lorentz factors $\gamma$ can be estimated from the fact that the synchrotron radiation is emitted at a frequency $\gamma^{2} v_{\mathrm{B}}$, where $v_{\mathrm{B}}$ is the cyclotron frequency. For a synchrotron peak in the IR, at $v=10^{14} \mathrm{~Hz}$, we have $\gamma^{2} B_{\mathrm{kG}}=3.6 \times 10^{4}$, where $B=B_{\mathrm{kG}} \mathrm{kG}$ is the magnetic field in the emission region. An upper limit on $B$ can be placed by constraining the emission at $231 \mathrm{GHz}$ to be optically thin. Then, $B \lesssim 250 R_{11}^{4} \mathrm{G}$, where $R=10^{11} R_{11} \mathrm{~cm}$ is the source radius, and $\gamma \gtrsim 400 R_{11}^{-2}$. Optically thin emission implies a brightness temperature below the equipartition value of $\sim 10^{11} \mathrm{~K}$ (Readhead 1994), which in turn implies $R_{11} \gtrsim 1=7 r_{\mathrm{g}}$, where $r_{\mathrm{g}}$ is specified for a $10^{5} \mathrm{M}_{\odot} \mathrm{IMBH}$. The $34 \mathrm{GHz}$ upper limit implies that the SED must transition to optically thick emission between 34 and $231 \mathrm{GHz}$, implying $R_{11} \lesssim 1.3$ at $34 \mathrm{GHz}$. Depending on the transition frequency between optically thick and thin emission, and the intervening absorbing column, detectable IC radiation may be expected in the far-UV to X-ray band. We encourage a search for such emission.

\subsection{A thermal interpretation}

The alternative hypothesis for CO-0.40- $0.22 *$ is that the radio emission detected by Oka et al. (2017) is thermal in nature. The best possibility is blackbody emission from warm dust. A hyper-compact $\mathrm{HII}$ region (Kurtz 2005) is unlikely, because the $32-266 \mathrm{GHz}$ spectral index constraint would imply optically thick emission at $266 \mathrm{GHz}$ that with a canonical $\mathrm{HII}_{\text {II }}$ region brightness temperature of $8000 \mathrm{~K}$ implies an amazingly small angular size of 5 mas (40 au at $\mathrm{Sgr}$ A*). Additionally, hot molecular cores (Kurtz et al. 2000) and cold cores (Rathborne, Jackson \& Simon 2006) are not viable interpretations for CO-0.40-0.22*. Hot cores are often associated with $\mathrm{H}_{2} \mathrm{O}$ maser emission and molecular outflows, neither of which are observed in CO-0.40-0.22* (SIMBAD; Oka et al. 2017), and the size of CO- $0.40-0.22 *(\lesssim 0.04 \times[D /(8.3 \mathrm{kpc})] \mathrm{pc}$ from the ALMA observations) is smaller than expected for cold cores.

Warm $(\sim 20-60 \mathrm{~K})$ dust grains in protostellar discs can result in $30-300 \mathrm{GHz}$ spectral indices in the range $\alpha \sim 2-2.5$ (Scaife et al. 2010). In this scenario, the ALMA-detected flux density can be used to estimate the mass of the circumstellar material (dust and gas). Following Beckwith et al. (1990), temperatures in the range $\sim 20$ $60 \mathrm{~K}$ give disc masses in the range $1.2-4.4 M_{\odot} \times[D /(8.3 \mathrm{kpc})]^{-2}$. Such discs are common around young massive stars (e.g. Jiang et al. 2005). The implied UV continuum from the central stellar object may create an HII region that dominates the radio spectrum at lower frequencies (Zapata et al. 2006). The dust disc would be transparent to IR emission, implying that the IR detections of CO-0.40-0.22* may be due to the central stellar object. Observations at frequencies $>266 \mathrm{GHz}$ should reveal a spectrum that rises up to the dust thermal peak at a few terahertz, and observations at $<32 \mathrm{GHz}$ may reveal an HII region. 


\section{A POSITIONALLY COINCIDENT IR-DARK CLOUD}

In our inspection of Spitzer/IRAC images at the position of CO-0.40$0.22 *$, we found an arrowhead-shaped region of high extinction against the diffuse Galactic mid-IR background and stellar field. This region (Fig. 3), which we term the Freccia Rossa, is coincident in position with CO-0.40-0.22*, and is additionally comparable in position and extent to the high-velocity compact molecular cloud CO-0.40-0.22. The Freccia Rossa has previously been identified as an IR-dark cloud (IRDC) in near-IR (DC 6; Nagayama et al. 2009) and mid-IR data (MSXDC G359.60-0.22; Simon et al. 2006). The Freccia Rossa is also detected in (thermal) emission in the $1.1 \mathrm{~mm}$ continuum Bolocam Galactic Plane Survey (BGPS G359.62-0.24; Bally et al. 2010) with a comparable morphology to the extinction nebula. The inferred mass is $8 \times 10^{3}-3 \times 10^{4} \mathrm{M}_{\odot}$ (assuming a Galactic Centre distance).

The distance to the Freccia Rossa is uncertain. Based on an analysis of the near-IR stellar field obscured by the cloud, Nagayama et al. (2009) suggest a distance of $3.6 \mathrm{kpc}$ from the Earth. A smaller molecular cloud at $V_{\mathrm{LSR}}=15-25 \mathrm{~km} \mathrm{~s}^{-1}$ with two sites of star formation is also positionally consistent with the Freccia Rossa; maser spots at the position and velocity of this cloud have a parallaxbased distance measurement of $3.1 \mathrm{kpc}$ (Iwata et al. 2017). However, the larger CO-0.40-0.22 cloud is most likely within the Galactic $\mathrm{CMZ}$, given its broad velocity width and an associated narrow $\mathrm{CO}$ absorption feature consistent with the $3 \mathrm{kpc}$ Arm (Oka et al. 2012). Bally et al. (2010) contend that the Freccia Rossa IRDC is also most likely within the CMZ, given the detection statistics of $1.1 \mathrm{~mm}$ clumps in their survey. Further, its inferred gas column density of $N_{\mathrm{H}_{2}}=1.2 \times 10^{24} \mathrm{~cm}^{-2}$ (Bally et al. 2010), which is independent of distance, implies a CO J $=1-0$ line intensity of $\approx 6 \times 10^{3} \mathrm{~K} \mathrm{~km} \mathrm{~s}^{-1}$ (Bolatto, Wolfire \& Leroy 2013), which is high, but consistent with the measured intensity of CO-0.40-0.22 (Oka et al. 1998). Although the value of $\mathrm{N}_{\mathrm{H}_{2}}$ is significantly above the observed threshold for rapid star formation in the solar neighbourhood of $\sim 1.4 \times 10^{22} \mathrm{~cm}^{-2}$ (Lada, Lombardi \& Alves 2010), only two star-forming cores may be associated with the Freccia Rossa if it is at $\sim 3 \mathrm{kpc}$ (Nagayama et al. 2009), and none if it is in the CMZ. This is consistent with a CMZ location, where the higher ambient pressure may increase the critical density for gravitational collapse in a turbulent environment by a factor of $\sim 10^{4}$ (Rathborne et al. 2014).

We are therefore motivated to consider the possibility that CO$0.40-0.22$ is physically associated with the Freccia Rossa, and that the system is within the CMZ. The physical association of CO0.40-0.22 and the Freccia Rossa would imply a surprisingly large velocity of $V_{\mathrm{LSR}} \approx-70 \mathrm{~km} \mathrm{~s}^{-1}$ for the system (Oka et al. 1998; Tanaka et al. 2014). This velocity is likely systemic relative to the Galactic rotation because of the Galactic-centre sightline.

\subsection{Radio emission on large angular scales}

We searched our ATCA observations for emission on larger angular scales associated with the Freccia Rossa system. Upon making an image of our data in both the $32.5 \mathrm{GHz}$ and $36 \mathrm{GHz}$ sidebands with a ultravioletUV taper corresponding to an image-plane Gaussian of $35 \operatorname{arcsec}$ FWHM(1/3 of the primary beam FWHM) and a robust parameter of 0.5 , a resolved source of $1.5 \pm 0.2 \mathrm{mJy}$ peak flux density was detected at a position coincident with CO-0.40-0.22. This source was however poorly characterized by our observations, as only a few baselines were sensitive to this angular scale. We therefore searched the ATCA archive for observations at this loca- tion, and found $24.6 \mathrm{GHz}$ continuum data obtained with an identical $\mathrm{CABB}$ setup, but in the more compact $\mathrm{H} 75$ configuration, as part of the C2927 project. Three pointings of a mosaic, totalling $580 \mathrm{~s}$ of integration time, were observed near the position of CO-0.40-0.22. We calibrated and imaged each pointing as described above, except that the long baselines to antenna 6 were excluded as they were not possible to calibrate. The three images were then summed with primary beam weighting to produce a mosaic image at the position of CO-0.40-0.22 (Fig. 4).

An extended source with an FWHM of $40 \times 80$ arcsec is detected at a position coincident with CO-0.40-0.22, with a peak flux density of $8.3 \pm 0.4 \mathrm{mJy}$. The spectral index of this emission between these $24.6 \mathrm{GHz}$ data and the $34.25 \mathrm{GHz}$ detection is difficult to estimate, because much of the emission is on angular scales larger than that are probed by the $34.25 \mathrm{GHz}$ data. The source is none the less likely to be the thermal brehmsstrahlung emission from an HII region, as it is too bright to correspond to the dust blackbody emission detected at $1.1 \mathrm{~mm}$ (Bally et al. 2010). Synchrotron emission from, for example, a supernova remnant is unlikely given the association with and comparable angular scales of the emission and CO-0.400.22 . In addition, no coincident emission is observed at $150 \mathrm{MHz}$ with a comparable angular resolution in the TIFR GMRT Sky Survey (TGSS; Intema et al. 2017), with a $3 \sigma$ upper limit of $45 \mathrm{mJy}$. This implies a $150 \mathrm{MHz}-24.6 \mathrm{GHz}$ spectral index of $\alpha>-0.33$ thatis flatter than most radio supernova remnants (Dubner \& Giacani 2015). This is, however, consistent with an HII region. The comparable angular sizes of the CO-0.40-0.22 molecular gas cloud and the candidate HII region imply that the HII region cannot be embedded within the cloud. However, the required incident rate of Lyman-continuum photons, which we estimate following Kerton, Arvidsson \& Alexander (2013) to be $\sim 10^{47} \mathrm{ph} \mathrm{s}^{-1}$, is consistent with the mean ambient UV photon energy density in the CMZ (e.g. Rodríguez-Fernández et al. 2004). We interpret the candidate HII region as corresponding to the photodissociation region of the molecular gas cloud in the Freccia Rossa, and not as an indication of internal star formation.

\section{DISCUSSION}

We first address the arguments made by Oka et al. (2016) and Oka et al. (2017) for the presence of an IMBH associated with the CO-0.40-0.22 cloud. Our data do not exclude a synchrotron origin for the ALMA-detected emission from CO-0.40-0.22*, but tension exists with Sgr A*-like models. However, the presence of an IR counterpart, and our lower limit on the radio spectral index, makes a protostellar-disc interpretation of $\mathrm{CO}-0.40-0.22 *$ plausible.

If $\mathrm{CO}-0.40-0.22 *$ is not an IMBH, what could cause the large spread of velocities in CO-0.40-0.22? Of the explanations considered by Tanaka et al. (2014), the possibility of an expanding molecular shell driven by multiple supernovae is least impacted by our work. CO-0.40-0.22 lies on the edge of a large molecular shell (shell 1 of Tanaka et al. 2014). However, if the large systemic velocity of CO-0.40-0.22 matches the systemic velocity of the Freccia Rossa cloud, none of the mechanisms suggested by Tanaka et al. (2014) are feasible. This is because the total mass of $\sim 3 \times 10^{6} \mathrm{M}_{\odot}$ and the systemic velocity of $70 \mathrm{~km} \mathrm{~s}^{-1}$ together imply an exceedingly large kinetic energy of $\sim 10^{53} \mathrm{erg}$.

\subsection{A plunging halo object?}

The Milky Way's bar is believed to be responsible for many of the highly non-circular velocities seen in clouds in the CMZ (Liszt 


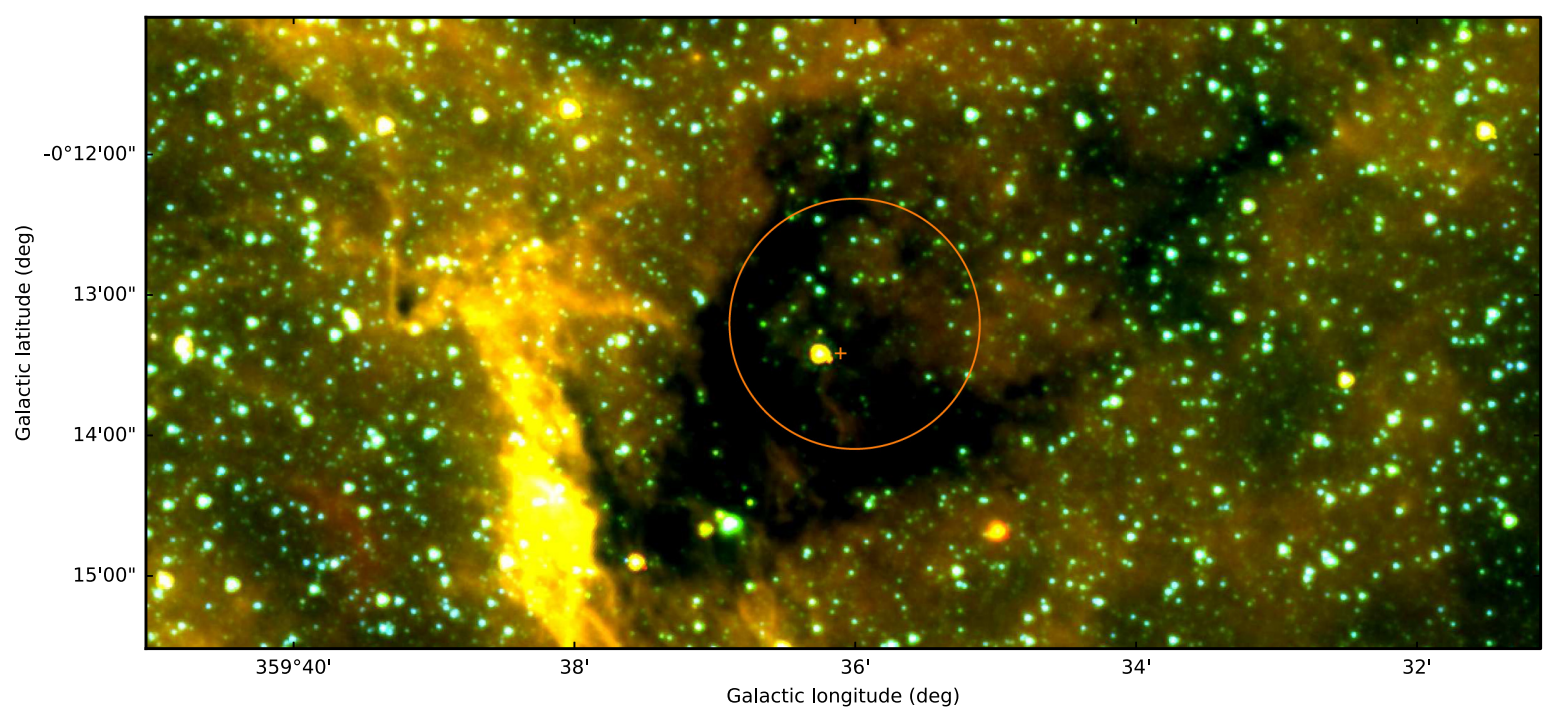

Figure 3. Three-colour mid-IR image of the Freccia Rossa IRDC from the Spitzer GLIMPSE data. The red, green, and blue components correspond to the channels $4(8 \mu \mathrm{m}), 3(5.8 \mu \mathrm{m})$, and $1(3.6 \mu \mathrm{m})$ of the IRAC instrument, respectively. The cross indicates the position of the IMBH candidate CO-0.40-0.22*, and the circle indicates the position and extent of the CO-0.40-0.22 high-velocity compact cloud (Tanaka et al. 2014; Oka et al. 2017).

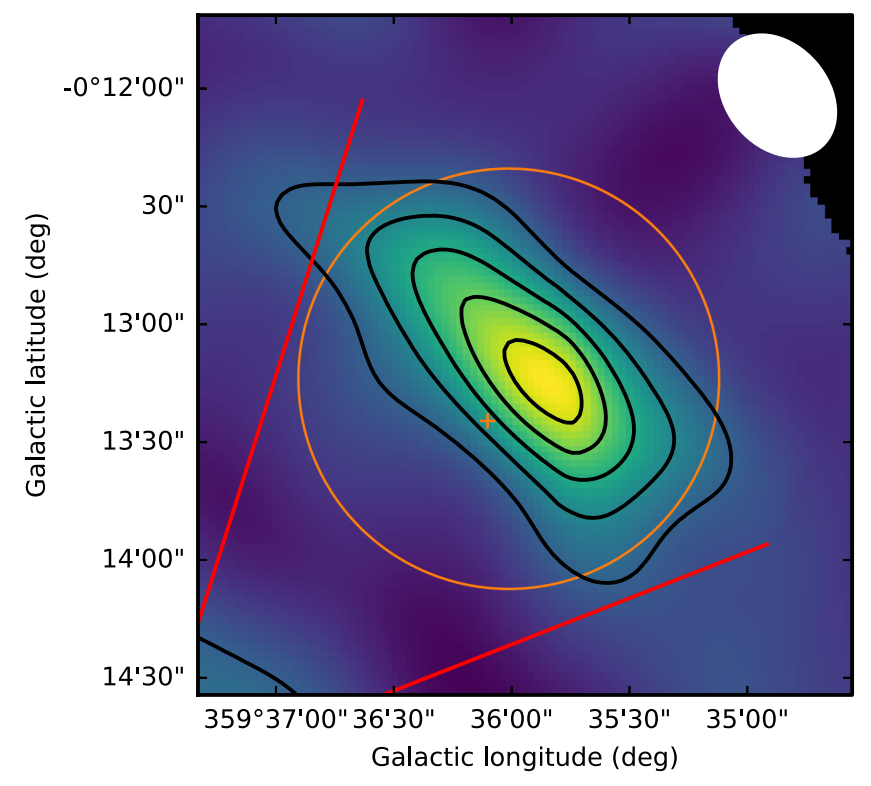

Figure 4. ATCA radio-continuum mosaic image of the CO-0.40-0.22 molecular cloud at a frequency of $24.6 \mathrm{GHz}$. The contours are at -1.5 (dashed black), 1.5, 3.0, 4.5, 6.0, and $7.5 \mathrm{mJy}$ (solid black). The orange cross indicates the position of $\mathrm{CO}-0.40-0.22 *$, and the orange circle indicates the position and extent of CO-0.40-0.22 (Tanaka et al. 2014; Oka et al. 2017). The synthesized beam FWHM is indicated by a solid white ellipse in the top-right corner. The red wedge illustrates the approximate position of the Freccia Rossa cloud.

2008; Ridley et al. 2017) such as the Freccia Rossa. The high dispersion and radial velocity of the Freccia Rossa is reminiscent of those seen in simulations of molecular flow in the bar potential (Sormani et al. 2017). However the high velocity perpendicular to the disc implied by the morphology of the Freccia Rossa is not expected in such models, although there are other observational indications of a tilt in the bar (Liszt 2008).

A compelling hypothesis for the Freccia Rossa is that of a highvelocity cloud (HVC) from the Galactic halo plunging into the
Milky Way disc. HVCs detected in Hi line emission, with masses $>10^{6} \mathrm{M}_{\odot}$ and velocities $V_{\mathrm{LSR}}>90 \mathrm{~km} \mathrm{~s}^{-1}$, have long been observed to populate the Milky Way halo (Wakker \& van Woerden 1997). Cold $(\sim 10 \mathrm{~K})$ dust has also recently been associated with HVCs (Miville-Deschênes et al. 2005). Although little evidence exists for HVCs that have impacted the Milky Way disc, simulations (e.g. Kereš \& Hernquist 2009) suggest that clouds with masses $\gtrsim 3 \times 10^{5} \mathrm{M}_{\odot}$ should survive the fall through the inner halo of the Galaxy. Recently, Park et al. (2016) associated an HVC with a supershell of $\mathrm{H}_{\mathrm{i}}$ in the outskirts of the Galactic disc.

Under this hypothesis, the dynamics of the molecular cloud CO$0.40-0.22$ and its high systemic velocity, which we associate with the Freccia Rossa, are explained by the bulk motion of the system and the ensuing interaction with the Milky Way interstellar medium. The cometary morphology of the Freccia Rossa, with the densest dust to be found at the tip of the cloud (Bally et al. 2010), provides circumstantial evidence for this scenario. We then have no extrinsic reason to interpret the compact source CO-0.40-0.22* as an IMBH; a protostellar disc is an alternative possibility. Detailed imaging and modelling of the dust continuum and molecular-line emission will help unravel the dynamics of the Freccia Rossa system.

\section{CONCLUSIONS}

We examine the evidence for an IMBH interpretation of the highvelocity compact molecular cloud CO-0.40-0.22 (Oka et al. 2016), and the associated millimetre-continuum source $\mathrm{CO}-0.40-0.22 *$ (Oka et al. 2017). The broad-band SED of CO-0.40-0.22*, with its steep $(\alpha>1.77)$ radio spectrum and IR counterpart, is in tension with Sgr A*-like models despite the comparably underluminous nature assuming a $10^{5} \mathrm{M}_{\odot}$ accreting object. Although we cannot exclude a synchrotron origin for the emission, we show that the system is also consistent with emission from a protostar surrounded by a $1.2-4.4 \mathrm{M}_{\odot} \times[D /(8.3 \mathrm{kpc})]^{-2}$ disc. Further broad-band measurements of the SED will establish the nature of CO-0.40-0.22*.

We also find that CO-0.40-0.22(*) is positionally coincident with an arrowhead-like IRDC with a total mass of up to $\sim 3 \times 10^{6} \mathrm{M}_{\odot}$ (the Freccia Rossa). If the $V_{\mathrm{LSR}} \approx-70 \mathrm{~km} \mathrm{~s}^{-1}$ systemic velocity of 
CO-0.40-0.22 is consistent with that of the Freccia Rossa cloud, we hypothesize that the system is the remnant of an HVC that plunged into the Milky Way from the Galactic halo. This scenario may account for the kinematics of the CO-0.40-0.22 molecular cloud, leaving no extrinsic reason to invoke an associated IMBH.

\section{ACKNOWLEDGEMENTS}

We thank the referee for helping in improving the presentation of the manuscript, and Mattia Sormani for pointing out the possibility of bar-induced motions. We are grateful to the staff of Commonwealth Scientific and Inductrial Research Organisation (CSIRO) Astronomy and Space Science for rapidly scheduling our observations. The Australia Telescope Compact Array is a part of the Australia Telescope National Facility that is funded by the Australian Government for operation as a National Facility managed by CSIRO. ESP's research was funded in part by the Gordon and Betty Moore Foundation through grant GBMF5076. This work is based in part on observations made with the Spitzer Space Telescope, which is operated by the Jet Propulsion Laboratory, California Institute of Technology under a contract with National Aeronautics and Space Administration. This research has made use of the SIMBAD data base, operated at Centre de donees astronomiques de Strasbourg, Strasbourg, France.

\section{REFERENCES}

Bally J. et al., 2010, ApJ, 721, 137

Beckwith S. V. W., Sargent A. I., Chini R. S., Guesten R., 1990, AJ, 99, 924

Bolatto A. D., Wolfire M., Leroy A. K., 2013, ARA\&A, 51, 207

Churchwell E. et al., 2009, PASP, 121, 213

Colpi M., 2014, Space Sci. Rev., 183, 189

Cross N. J. G. et al., 2012, A\&A, 548, A119

Dubner G., Giacani E., 2015, A\&AR, 23, 3

Genzel R., Eisenhauer F., Gillessen S., 2010, Rev. Mod. Phys., 82, 3121

Gillessen S., Eisenhauer F., Fritz T. K., Pfuhl O., Ott T., Genzel R., 2013, in de Grijs R., ed., Proc. IAU Symp. Vol. 289, Advancing the Physics of Cosmic Distances, Cambridge Univ. Press, Cambridge, p. 29

Gilmore G., Wyse R. F. G., Norris J. E., 2002, ApJ, 574, L39

Greene J. E., 2012, Nat. Commun., 3, 1304

Intema H. T., Jagannathan P., Mooley K. P., Frail D. A., 2017, A\&A, 598, A78

Iwata Y., Kato H., Sakai D., Oka T., 2017, ApJ, 840, 18

Jiang Z., Tamura M., Fukagawa M., Hough J., Lucas P., Suto H., Ishii M., Yang J., 2005, Nature, 437, 112

Kereš D., Hernquist L., 2009, ApJ, 700, L1

Kerton C. R., Arvidsson K., Alexander M. J., 2013, AJ, 145, 78

Kurtz S., 2005, in Cesaroni R., Felli M., Churchwell E., Walmsley M., eds, Proc. IAU Symp. Vol. 227, Massive Star Birth: A Crossroads of Astrophysics. Cambridge Univ. Press, Cambridge, p. 111
Kurtz S., Cesaroni R., Churchwell E., Hofner P., Walmsley C. M., 2000, Protostars and Planets IV, Univ. Arizona Press, Tucsan, AZ

Lada C. J., Lombardi M., Alves J. F., 2010, ApJ, 724, 687

Liszt H. S., 2008, A\&A, 486, 467

Mahadevan R., 1997, ApJ, 477, 585

Minniti D. et al., 2010, New Astron., 15, 433

Miville-Deschênes M.-A., Boulanger F., Reach W. T., Noriega-Crespo A., 2005, ApJ, 631, L57

Nagayama T. et al., 2009, PASJ, 61, 283

Oka T., Hasegawa T., Sato F., Tsuboi M., Miyazaki A., 1998, ApJS, 118, 455

Oka T., Mizuno R., Miura K., Takekawa S., 2016, ApJ, 816, L7

Oka T., Nagai M., Kamegai K., Tanaka K., Kuboi N., 2007, PASJ, 59, 15

Oka T., Onodera Y., Nagai M., Tanaka K., Matsumura S., Kamegai K., 2012, ApJS, 201, 14

Oka T., Tsujimoto S., Iwata Y., Nomura M., Takekawa S., 2017, Nat. Astron., 1,709

Özel F., Psaltis D., Narayan R., 2000, ApJ, 541, 234

Park G., Koo B.-C., Kang J.-h., Gibson S. J., Peek J. E. G., Douglas K. A., Korpela E. J., Heiles C. E., 2016, ApJ, 827, L27

Rathborne J. M., Jackson J. M., Simon R., 2006, ApJ, 641, 389

Rathborne J. M. et al., 2014, ApJ, 795, L25

Readhead A. C. S., 1994, ApJ, 426, 51

Rees M. J., Begelman M. C., Blandford R. D., Phinney E. S., 1982, Nature, 295, 17

Ridley M. G. L., Sormani M. C., Treß R. G., Magorrian J., Klessen R. S., 2017, MNRAS, 469, 2251

Rodríguez-Fernández N. J., Martín-Pintado J., Fuente A., Wilson T. L., 2004, A\&A, 427, 217

Sault R. J., Teuben P. J., Wright M. C. H., 1995, in Shaw R. A., Payne H. E., Hayes J. J. E., eds, ASP Conf. Ser. Vol. 77, Astronomical Data Analysis Software and Systems IV. Astron. Soc. Pac., San Francisco, p. 433

Scaife A. M. M. et al., 2010, MNRAS, 403, L46

Simon R., Jackson J. M., Rathborne J. M., Chambers E. T., 2006, ApJ, 639, 227

Sormani M. C., Tress R. G., Ridley M., Glover S. C. O., Klessen R. S., Binney J., Magorrian J., Smith R., 2017, MNRAS, 475, 2383

Tanaka K., Oka T., Matsumura S., Nagai M., Kamegai K., 2014, ApJ, 783, 62

van Loon J. T. et al., 2003, MNRAS, 338, 857

Volonteri M., 2010, A\&AR, 18, 279

Wakker B. P., van Woerden H., 1997, ARA\&A, 35, 217

Wilson W. E. et al., 2011, MNRAS, 416, 832

Wolfe B., Melia F., 2006, ApJ, 637, 313

Yuan F., Quataert E., Narayan R., 2003, ApJ, 598, 301

Zapata L. A., Rodríguez L. F., Ho P. T. P., Beuther H., Zhang Q., 2006, AJ, 131,939

This paper has been typeset from a $\mathrm{T}_{\mathrm{E}} \mathrm{X} / \mathrm{L} \mathrm{T} \mathrm{E} \mathrm{X}$ file prepared by the author. 